\title{
Some results and applications using fuzzy logic in artificial intelligence
}

\author{
Irene Díaz ${ }^{1}$, Anca L. Ralescu ${ }^{2}$, Dan A. Ralescu ${ }^{3}$, and Luis J. \\ Rodríguez-Muñiz ${ }^{4}$
}

\author{
Algunas veces encuentras en la vida \\ una amistad especial: \\ ese alguien que al entrar en tu vida \\ la cambia por completo. \\ M. Benedetti, "Algunas amistades"
}

\begin{abstract}
In this chapter, several results and applications in artificial intelligence based on fuzzy logic are presented. We aim to highlight some recent works that are connected with the topic of this book, both in the theoretical and in the applied fields.
\end{abstract}

\section{Introduction}

Pedro Gil was one of the frontrunners of information theory in Spain [13], many of his works were devoted to this topic. Apart from his pioneering work in information theory, in the 80's he established links with uncertainty and imprecision by exploring research topics related to fuzzy sets and fuzzy logic. One of the first connections he established focused on the need of aggregating information; t-norms and t-conorms become a useful tool for some situations related to fuzzy measures and fuzzy integrals (e.g. [12, 38, 15, 17]). These are the topics we choose to cover in this memorial article dedicated to our beloved teacher, colleague, and friend. In particular, for two of the authors, Irene Díaz and Luis J. Rodríguez-Muñiz, this is a very special work, since they were both students in Pedro Gil's course on Information Theory at the Faculty of Sciences in the University of Oviedo. But the other two authors, Dan Ralescu and Anca Ralescu, had also the opportunity to meet Pedro Gil

\footnotetext{
${ }^{1}$ Universidad de Oviedo (Spain) sirene@uniovi.es . ${ }^{2}$ University of Cincinnati (USA) anca.ralescu@uc.edu . ${ }^{3}$ University of Cincinnati (USA) dan.ralescu@uc.edu .

${ }^{4}$ Universidad de Oviedo (Spain) luisj@uniovi.es
} 
in their first contacts with the University of Oviedo, more than twenty years ago. We all cherished Pedro as a friend, a colleague, and a master.

The structure of this chapter is divided into six sections. Section 1 corresponds to the introduction. Sections 2 to 4 are mainly focused on theoretical resultas while Section 5 draws some applications. Finally, some concluding remarks are outlined.

\section{Aggregation Operators}

Aggregation operators are very useful tools in many fields when the need of combining different values or information inputs arises [2]. Mainstream research approaches are based on the study of theoretical properties of aggregation operators, as well as the analysis of different values when the operator is depending on a parameter.

In [39] a new approach to the study of aggregations functions, based not only on the theoretical properties, but on the statistical behavior of the outputs was initiated. A procedure to carry out statistical comparisons between outputs of different aggregation functions was adopted, which was used to compare several $t$-norms (minimum, product, and Lukasiewicz), as well as some means (geometric, arithmetic, and quadratic). Based on a large number of simultations, the conditions under which outputs of aggregations are distinguishable (or not) were studied. Hence, the focus for practitioners moves from the theoretical issues to the practical behavior of results.

The procedure introduced in [39] was further pursued in [41], where comparisons were made for parametric $t$-norms. The goal in this work was to provide simple rules that can be used for selecting the parameter value. A strong graphic support was introduced to help visualize the similarities and differences between $t$-norm outputs. Also, the effects of the $t$-norm arity, the sample size, the input distribution were analyzed. The main result of this work is the identification of different combinations of conditions for which, from a practical point of view, the choice of the parameter value is irrelevant.

In addition, in [43] properties related to Dujmovic's Iterative OWA operator were studied. Iterative OWA (ItOWA) operators as proposed by Dujmovic, is a two-stage procedure for computing the weighting vector by a double nested iteration: (i) weights at step $h$ are computed as limit to infinity of a matrix power, (ii) the result is used to start the computation at step $h+1$, until the OWA operator arity $n$ is reached. [43] proposes an analytical solution to this procedure. This theoretical result enables a faster computation of the weighting vector and characterization in terms of weights values, attitudinal character and entropy.

Ralescu et al. in [31] studies the concept of optimal aggregation as it plays an important role towards developing a theory of aggregation of fuzzy concepts, based on non-additive set-functions and nonlinear (Choquet or fuzzy) 
integrals. A framework supporting such aggregations, that are useful to decision making based on distributed sources of evidence is developed. In [46] arithmetic operations for LR mixed fuzzy random variables commonly used in practice for modeling fuzzy stochastic phenomena are studied. The operations are proposed based on mean chance measure, which as a natural extension of both the probability of a random event and the credibility of a fuzzy event, measures the mean or expected (in the sense of probability) credibility that the fuzzy random event occurs. [30] studies the equivalence between aggregation of fuzzy sets and integration with respect to non additive set functions. The concepts of co-monotonic maxitivity is a more desirable requirement than co-monotonic additivity.

The aggregation of rankings is a recurrent task in several applications, especially in the context of social choice theory. In this framework [19] has studied some ranking aggregation strategies to represent group's opinion. In particular the strength with which $a \geq b \geq c$ is supported, should not be less than both the strength with which $a \geq b$ and $b \geq c$ are supported. A first approach to this ranking rule considering totally specified monotone reciprocal relations on a bipolar qualitative scale has already been taken. In this paper, a more general setting is considered: each voter is allowed to provide a partially specified reciprocal relation (that may not be monotone) on the unit interval. In this work we have also explored new ways of measuring the cost of imposing monotonicity are introduced. In [19], a more general setting is considered: each voter is allowed to provide a partially specified reciprocal relation (that may not be monotone) on the unit interval. Also explored are new ways of measuring the cost of imposing monotonicity. In [17] different representations of votes (the votrix and the votex) are introduced. The former is a formalization of the well-known reciprocal matrix of pairwise comparisons between candidates already introduced by Condorcet. The latter is an extension of this reciprocal matrix considering hitherto unexploited information. These two representations lead to two monotonicity-based ranking rules.

In [18] we study an aspect of monotonicity that complements previous studies. In particular, it is studied if there is a true ranking on the set of candidates and every voter expresses a ranking on the set of candidates, then the number of times that each ranking is expressed should decrease when we move away from this true ranking in terms of pairwise discordances. In addition, we propose a probabilistic model that allows to formulate the choice of the best ranking as a maximum likelihood estimation problem.

\section{Extension of Fuzzy Sets}

Some extensions of the standard fuzzy sets have been extensively studied during the last 20 years. Interval, Atanassov's intuitionistic or hesitant fuzzy sets are representative examples of these extensions. In [21] an ordering frame- 
work required to work with interval-valued hesitant fuzzy sets is presented. In fact, ordering sets is a long-standing open problem due to its remarkable importance in many areas such as decision making, image processing or human reliability. Methods for ordering finitely generated sets as a generalization of those methods previously defined for ordering intervals were introduced in [21]. In addition, these orderings between finitely generated sets are also improved to present ordering between finite interval-valued hesitant fuzzy sets.

Finally, finite interval-valued hesitant fuzzy preference relations are introduced and used to define a new order between finite interval-valued hesitant fuzzy sets. Along the same lines, in [26] some concepts related to partitioning for interval-valued fuzzy sets is studied. Partitioning is a long-standing open problem due to its remarkable importance in many areas such as clustering. The definition of this partitioning method involves a definition of an ordering relation for finite interval-valued fuzzy sets membership degrees, i.e., finitely generated sets, as well as the definitions of t-norm and t-conorm for these kinds of sets.

Still focusing on interval-valued fuzzy sets, in [25] a definition of entropy for an interval-valued hesitant fuzzy environment is provided. As the properties of this kind of sets are more complex, the entropy is built by three different functions, where each one represents a different measure: fuzziness, lack of

knowledge, and hesitance. Using all, an entropy measure for interval-valued hesitant fuzzy sets is obtained, quantifying various types of uncertainty.

Yang et al. [45] introduces the definition of type-2 uncertain variables within the framework of uncertainty theory through introduction of generalized uncertain measures and focuses on more complex twofold uncertainties. Some uncertainty reduction methods associated with type- 2 uncertain variables are also proposed for convenience of applicability, including reduction of optimistic value, pessimistic value and expected value. Moreover, four classes of type- 2 uncertain variables are reduced to type- 1 uncertain variables with specific uncertainty distributions.

\section{Classification}

Going from aggregation operators to a broader knowledge domain, as artificial intelligence is, we have also used fuzzy logic to extend crisp classification algorithms. In particular, in [33] the fuzzy extension of a previous crisp measure is introduced. The implementation of this measure into an algorithm proved to improve results obtained with similar algorithms, as well as it introduced and efficient tool to deal with imprecision in human judgments.

Extracting rules from databases is one of the main tasks in artificial intelligence. Different algorithms for learning rules both from crisp and fuzzy databases have been proposed in the literature. This way, learning informa- 
tion from databases is opposed to general policies about data protection. Thus, statistical disclosure control is a paradigm consisting of finding the optimal balance between releasing statistical data and protecting confidentiality of sensitive information.

Fuzzy logic is a useful tool in finding this balance, since fuzzy association rules can provide interpretable rules while protecting sensitive records in the database. In [40] this problem was investigated with the aim of establishing the existence of a set of rules able to break protection, assessing the interpretability of the model and identifying the ability of attributes for revealing sensitive information. The results showed that fuzzy rules are, in general, simpler and easier to interpret than others.

Based on the results obtained in [40] a new approach for dealing with fuzzy sets in data protection was developed in [11]. In particular, the notion of fuzzy cardinality becomes a key idea in [40]: counting the number of elements in a given class is the basis to determine if data of individuals within that class could be disclosed. The type of fuzzy cardinality used in this work is the simplest $\sigma$-count, being an open problem how to compute the cardinality with more interpretable definitions of fuzzy counting as those in [29, 27].

Following this path, in $[23,24]$ fuzzy notions were introduced in wellknown measures in the privacy framework as $k$-anonymity, $l$-diversity and $t$-closeness. These works propose the extension of these three measures when the data are protected using fuzzy sets instead of intervals or representative elements. This methodology was tested using different fuzzy partition methods, obtaining an improvement in protecting data encoded using fuzzy sets. In addition, [10] provides a brief overview of the emerging research in privacy issues in social networks.

With regard to classification, [32] proposes a classification algorithm which considers explicitly geometric and statistical characteristics of the data and combines them into a class representation. The obtained method shows that the proposed algorithm is less sensitive to the training data set than other classifiers, which is an important property for a classification algorithm.

\section{Applications}

This section describes some of our recent results concerning applications of fuzzy logic to different knowledge domains, further from mathematics, statistics or theoretical artificial intelligence. The main novelty of these applications consists in introducing fuzzy logic, in many cases for the first time, in areas usually considered the domain of crisp techniques, demonstrating how fuzzy logic based procedures perform better than their crisp counterparts.

One important application of fuzzy sets focused on Artificial Vision. It has been initially developed for binary and gray scale images. Nevertheless, the color is an important source of information. For this reason, during the 
last years these techniques have been developed for color images. However, nowadays, the representation and the treatment of color images are still open problems [1].

Mathematical Morphology is the natural area for a rigorous formulation of many problems in image analysis, as well as a powerful non-linear technique which includes operators for the filtering, texture analysis, shape analysis, edge detection or segmentation. In the eighties, Matheron and Serra [14, 37] proposed the latest mathematical formulation of morphology within the algebraic framework of the lattices. This means that the definition of morphological operators needs a totally ordered complete lattice structure. In that context, before defining the basic morphological operators (erosion and dilation) it is necessary to define an order on the space used for processing the images.

Fuzzy Mathematical Morphology [5, 44] is an extension of the Mathematical Morphology's binary operators to gray level images, by redefining the set operations as fuzzy set operations. In $[7,8,9]$ we define the operators of the Fuzzy Mathematical Morphology for color images through the use of a fuzzy order. Other important works related to image processing are highlighted: In [16] information about spatial organization in an image is considered to improve object recognition and scene analysis tasks. Also, in [6] directional relative position relations are considered since they provide an important information about the spatial arrangement of objects in the scene. Such concepts are rather ambiguous, they defy precise definitions, but human beings have a rather intuitive and common way of understanding and interpreting them. Therefore in this context, fuzzy methods are appropriate to provide consistent definitions that integrate both quantitative and qualitative knowledge, thus providing a computational representation and interpretation of imprecise spatial relations, expressed in a linguistic way, and including quantitative knowledge. [6] reviews and compares different fuzzy approaches according to their properties and according to the types of questions they seek to answer.

Risk assessment in human reliability is another application area where fuzzy sets can provide solutions. Risk is usually mathematically formalized as a matrix that allows the classification of different kinds of errors according to their importance. This classification can help in decision making about the most important or urgent one. Usually the risk matrix takes into account only one criterion (most of the cases: economic impact). However, decision making in a company often considers more than one criterion. Therefore, it is interesting to consider at the same time more than one different risk matrix, each one associated with different criteria for consequences (for example, effects on people, environment, assets or reputation). In [22] we have developed a method to combine this information in order to classify the errors according to more than one criterion.

We have developed a general method to combine the information about different alternatives given by several experts or taking into account several 
criteria and the choice of the set of the best ones. This method can be applied in any environment where there exists interaction among the different alternatives or some experts are more reliable than others. This will be done by the definition of fuzzy preference relations and the use of different aggregation functions, in particular the weighted ordered weighted averaging operator.

Research in Recommendation Systems has been exponentially growing since the development of the Internet, more precisely, with the development of e-commerce. A Recommender System (RS) usually provides a rating or a preference for each user. To provide this recommendation an RS requires information about the preferences of the user in relation to the website (movies, books, songs, hotels, etc.). This information can be acquired explicitly by asking the users to rate items or implicitly by monitoring users' behavior (booked hotels or heard songs). RS can also use other kinds of information as demographic features (e.g., age, gender) or social information.

The research related to RS has been focused on movies, music and books recommendations, being music recommendation the most studied topic although, recently, it has been applied in other e-commerce domains. In [42] we face the problem of filtering from an e-shop catalog a set of products which might be interesting for the customer on the basis of preferences expressed by a group of users within a market segment. This problem is studied from a theoretical point of view by means of Dempster-Shafer Theory of Evidence (D-S Theory). The purpose of this work is to show how the D-S Theory can be used in the context of Recommendation Systems. In this work we propose to move from items to features in order to (i) reduce problem dimensionality and (ii) to infer user preferences even when they are not made explicit. Preferences induced by each feature are considered as an independent source of information, then combined by a rule. We also studied how to explore the subset inclusion lattice, once Belief and Plausibility are mapped over it. In addition, we outline some efficient algorithms to perform such an exploration.

Profile similarity is a key point in recommendation systems. In [34] it is studied how to assess the similarity between node profiles in a social network. Several approaches exist to study profile similarity, including semantic approaches and natural language processing. However, in this work we combine these aspects into a unified measure of profile similarity. Traditionally, semantic similarity is assessed using keywords, that is, formatted text information, with no natural language processing component. In this study we propose an alternative approach, whereby the similarity assessment based on keywords is applied to the output of natural language processing of profiles. A unified similarity measure results from this approach.

Ralescu et al. in [28] study the representation of an optimization with inexact constraints, as a fuzzy integral with respect to a capacity (or to an outer measure) rather than to a fuzzy measure. It is proved a mean-value theorem for the fuzzy integral, which has as a consequence the reduction of optimization with inexact constraints to classical optimization. Sufficient conditions for this reduction to hold are also provided in this work. 
Another recent field of application in which we have started to work is the use of artificial intelligence techniques for detecting and predicting dropout in higher education. This is a key problem for higher education institutions, and in the vast educational literature about it only classical statistical methods were present. In [36] we started a new line of research for considering other type of approaches, by applying our previous work on extracting rules from databases.

Finally, we also wanted to underline other recent research line because it connects two of Pedro's main beloved occupations: fuzzy logic and teaching. We have started an analysis of curricular guidelines in primary and secondary education (mainly focused on the Spanish framework) in order to determine how imprecision belongs to the so called "hidden curriculum". While uncertainty has been introduced in the analysis of the curricular standards in secondary school from the 80's, and in primary school from the beginning of 21st century, imprecision is used in many procedures: approximate and mental calculus, classification of events as sure, likely or unlikely, ranking events depending on their possibility, etc. We have started to study this type of situations from the educational point of view both for primary ([3]) and secondary school ([35]), and jointly ([4]) and, in a forthcoming work, we plan to develop experimental studies at schools.

\section{Concluding remarks}

We have collected different results recently achieved in our research lines with the aim of presenting connections of works developed by Pedro Gil in his multiple research interests. We are sure that Pedro Gil would like these works, and particularly he would appreciate applications in education, as the last one quoted, since he was always very concerned with teaching and learning processes not only at university level but also at previous educational stages. Most of all, our main goal was to pay a tribute to the researcher, the professor and, especially, the person of Pedro Gil. Recalling the poet, our beloved Pedro, we hope that in these pages we have attained singing the praises of "la madurez insigne de tu conocimiento"1.

\section{References}

1. Aptoula, E., Lefevre, S. (2008). On lexicographical ordering in multivariate mathematical morphology, Pattern Recognition Letters, 29 (2), 109-118.

2. Beliakov, G., Pradera, A., Calvo, T. (2007). Aggregation Functions: A Guide For Practitioners, Springer: Heidelberg.

${ }^{1}$ From "Llanto por Ignacio Sánchez-Mejías", Federico García Lorca, 1935. 
3. Blanco-Fernández, A., García-Honrado, I., Ramos-Guajardo, A.B., Rodríguez-Muñiz, L.J. (2014). Reflexiones sobre el tratamiento de lo inciertro en Educacin Primaria en Espaa. In Actas del XVII Congreso Espaõl sobre Tecnologías y Lógica Fuzzy, 573-578.

4. Blanco-Fernández, A., Díaz-Díaz, P., García-Honrado, I., Ramos-Guajardo, A.B., Rodríguez-Muñiz, L.J. (2016). A proposal for assessing imprecise concepts in Spanish primary and secondary schools, Int. J. Uncertainty, Fuzziness and Knowledge-Based Systems, 24 (Suppl. 2), 71-91.

5. Bloch, I., Maitre, H. (1993). Fuzzy Mathematical Morphology. Annals of Mathematical Artificial Intelligence 10(1-2), 55-84.

6. Bloch, I., Ralescu, A. (2003). Directional relative position between objects in image processing: a comparison between fuzzy approaches. Pattern Recognition 36 (7), 15631582.

7. Bouchet, A., Alonso, P., Díaz, I., Montes, S. (2017). On the performance of Some Edge Detectors for Gray Scale Images, Journal of Multiple-Valued Logic and Soft Computing, in press.

8. Bouchet, A., Alonso, P., Pastore, J.I., Díaz, I., Montes, S. (2016). Fuzzy Mathematical Morphology for color images defined by fuzzy preference relations, Pattern Recognition, 60, 720-733.

9. Bouchet, A., Quirós, P., Alonso, P., Ballarin, V., Díaz, I., Montes, S. (2015). Gray Scale Edge Detection using Interval-Valued Fuzzy Relations, International Journal of Computational Intelligence Systems, 8 (2), 16-27.

10. Díaz, I., Ralescu, A.L. (2012). Privacy Issues in Social Networks A Brief Survey. Communications in Computer and Information Science, 300, 509-518.

11. Díaz, I., Rodríguez-Muñiz, L.J., Troiano, L. (2012). Fuzzy sets in data protection: strategies and cardinalities, Logic Journal of the IGPL, 20,657-666.

12. Fernández, M.J., Suárez, F., Gil, P. (1993). T-eigen fuzzy sets. Information Sciences, 75 (1-2), 63-80.

13. Gil, P. (1981). Teoría matemática de la información. ICE: Madrid.

14. Matheron, G. (1975). Random sets and integral geometry. Willey: New York.

15. Miranda, P., Grabisch, M., Gil, P. (2000). Divergence measures and aggregation operators. Int. J. Uncertainty, Fuzziness and Knowledge-based Systems, 8 (6), 677-690.

16. Miyajima, K., Ralescu, A. (1994). Spatial organization in 2D segmented images: representation and recognition of primitive spatial relations. Fuzzy Sets and Systems 65 (2-3), 225-236.

17. Montes, S., Couso, I., Gil, P., Bertoluzza, C. (2002). Divergence measure between fuzzy sets. Int. J. Approximate Reasoning, 30 (2), 91-105.

18. Pérez-Fernández, R., Rademaker, M., Alonso, P., Díaz, I., Montes, S., De Baets, B. (2017). Monotonicity as a tool for differentiating between truth and optimality in the aggregation of rankings, Journal of Mathematical Psychology, 77, 1-9.

19. Pérez-Fernández, R., Rademaker, M., Alonso, P., Díaz, I., Montes, S., De Baets, B. (2017). Monotonicity-based ranking on the basis of multiple partially specified reciprocal relations, Fuzzy Sets and Systems, https://doi.org/10.1016/j.fss.2016.12.008.

20. Pérez-Fernández, R., Rademaker, M., Alonso, P., Díaz, I., Montes, S., De Baets, B. (2016). Representations of votes facilitating monotonicity-based ranking rules: from votrix to votex, International Journal of Approximate Reasoning, 73, 87-107.

21. Pérez-Fernández, R., Alonso, P., Bustince, H., Díaz, I., Jurío, A., Montes, S. (2015). Ordering finitely generated sets and finite interval-valued hesitant fuzzy sets, Information Sciences, 325, 375-392.

22. Pérez-Fernández, R., Alonso, P., Díaz, I., Montes, S. (2015). Multi-Factorial Risk AssessmentAn Approach based on Fuzzy Preference Relations,, Fuzzy Sets and Systems, $\mathbf{2 7 8}, 67-80$.

23. Quirós, P., Alonso, P., Díaz, I., Montes, S. (2014). On the Use of Fuzzy Partitions to Protect Data, Integrated Computer-Aided Engineering, 21, 355-366.

24. Quirós, P., Alonso, P., Díaz, I., Montes, S. (2015). Protecting data: A fuzzy approach, International Journal of Computer Mathematics, 92 (9), 1989-2000. 
25. Quirós, P., Alonso, P., Bustince, H., Díaz, I., Montes, S. (2015). An entropy measure definition for finite interval-valued hesitant fuzzy sets, Knowledge-Based Systems, 84, 121-133.

26. Quirós, P., Alonso, P., Díaz, I., Montes, S. (2016). On delta-epsilon partitions for Finite Interval-Valued Hesitant Fuzzy Sets, International Journal of Uncertainty, Fuzziness and Knowledge-Based Systems, 24 (2), 145-163.

27. Ralescu, D.A. (1995). Cardinality, quantifiers, and the aggregation of fuzzy criteria, Fuzzy Sets and Systems, 69, 355-365.

28. Ralescu, D.A., Ralescu, A.L. (2015). Optimization in a Fuzzy Environment. Libertas Mathematica (new series), 35 (2), 51-59.

29. Ralescu, A. (1986) A note on rule representation in expert systems. Information Sciences 38(2) 193-203.

30. Ralescu, A., Ralescu, D. (1997) Extensions of Fuzzy Agrregations. Fuzzy Sets and Systems 86 (3) 321-330.

31. Ralescu, A.L., Ralescu, D. A., Yamakata, Y. (2007). Inference by aggregation of evidence with applications to fuzzy probabilities. Information Sciences, 177 (2), 378-387.

32. Ralescu, A.L., Díaz, I., Rodríguez-Muñiz, L. J. (2015). A classification algorithm based on geometric and statistical information, Journal of Computational and Applied Mathematics, 275, 335-344.

33. Ranilla, J., Rodríguez-Muñiz, L.J. (2007). A heuristic approach to learning rules from fuzzy databases, IEEE Inteligent Systems, 22 (2), 62-68.

34. Rawashdeh, A., Rawashdeh, M., Díaz, I., Ralescu, A.L. (2012), Semantic Similarity between Nodes in a Social Network. Communications in Computer and Information Science 443, 76-85

35. Rodríguez-Muñiz, L.J., Díaz-Díaz, P. (2013). Imprecision, uncertainty and probability in Spanish secondary school: a working proposal. In Proceedings EUROFUSE 2013, 233-240.

36. Rodríguez-Muñiz, L. J., Bernardo, A., Esteban, M., and Díaz, I. (2017). University dropout: Discovering rules with machine learning methods, submitted.

37. Serra, J. (1982) Image analysis and mathematical morphology, Vol. I. Academic Press: London.

38. Suárez, F., Gil, P. (1986). Fuzzy expected value with semiconormed integrals. Trabajos de Estadística, 1 (1), 127-139.

39. Troiano, L., and Rodríguez-Muñiz, L.J. (2011). A statistical study of differences and similarities among aggregation functions, Logic Journal of the IGPL, 19 (2), 415-424.

40. Troiano, L., Rodríguez-Muñiz, L.J., Ranilla, J., and Díaz, I. (2012). Interpretability of fuzzy association rules as means of discovering threats to privacy, Int. J. Computer Mathematics, 89 (3), 325-333.

41. Troiano, L., Rodríguez-Muñiz, L.J., Marinaro, P., and Díaz, I. (2014). Statistical analysis of parametric t-norms, Information Sciences, 257, 138-162.

42. Troiano, L., Rodríguez-Muñiz, L.J., Díaz, I. (2015). Discovering User Preferences using Dempster-Shafer Theory. Fuzzy Sets and Systems, 278, 98-117.

43. Troiano, L., Díaz, I. (2016). An analytical solution to Dujmovic's Iterative OWA. International Journal of Uncertainty, Fuzziness and Knowledge-Based Systems, 24 (2), 165-179.

44. Vanegas, M.C., Bloch, I., Inglada, J. (2016). Fuzzy constraint satisfaction problem for model-based image interpretation. Fuzzy Sets and Systems, 286, 1-29.

45. Yang, L., Liu, P., Li, S. Gao, Y., Ralescu, D. A. (2015). Reduction methods of type-2 uncertain variables and their applications to solid transportation problem. Information Sciences, 291, 204-237.

46. Wang, K., Zhou, J., Ralescu, D.A. (2017). Arithmetic operations for LR mixed fuzzy random variables via mean chance measure with applications. Journal of Intelligent \& Fuzzy Systems 32 (1), 451-466. 